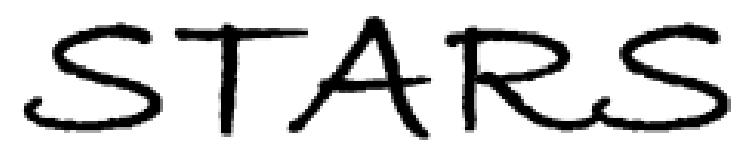

University of Central Florida

STARS

$1-1-1996$

\title{
Effects Of Mode Structure On Three-Dimensional Laser Heating Due To Single Or Multiple Rectangular Laser Beams
}

\author{
A. Kar \\ University of Central Florida \\ J. E. Scott \\ W. P. Latham
}

Find similar works at: https://stars.library.ucf.edu/facultybib1990

University of Central Florida Libraries http://library.ucf.edu

This Article is brought to you for free and open access by the Faculty Bibliography at STARS. It has been accepted for inclusion in Faculty Bibliography 1990s by an authorized administrator of STARS. For more information, please contact STARS@ucf.edu.

\section{Recommended Citation}

Kar, A.; Scott, J. E.; and Latham, W. P., "Effects Of Mode Structure On Three-Dimensional Laser Heating Due To Single Or Multiple Rectangular Laser Beams" (1996). Faculty Bibliography 1990s. 790.

https://stars.library.ucf.edu/facultybib1990/790

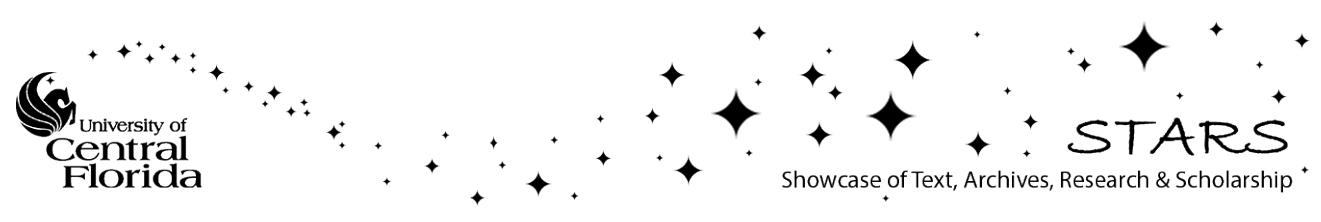




\section{Effects of mode structure on three- dimensional laser heating due to single or multiple rectangular laser beams}

Cite as: Journal of Applied Physics 80, 667 (1996); https://doi.org/10.1063/1.363555

Submitted: 09 November 1995 . Accepted: 08 April 1996 . Published Online: 04 June 1998

A. Kantor, J. E. Scott, and W. P. Latham

ARTICLES YOU MAY BE INTERESTED IN

Heat treating and melting material with a scanning laser or electron beam Journal of Applied Physics 48, 3895 (1977); https://doi.org/10.1063/1.324261

Thermal response of a laser-irradiated metal slab

Journal of Applied Physics 64, 3815 (1988); https://doi.org/10.1063/1.341388

Laser-induced surface heating

Journal of Applied Physics 73, 1066 (1993); https://doi.org/10.1063/1.353293

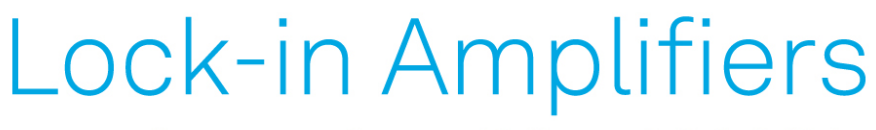

... and more, from DC to $600 \mathrm{MHz}$

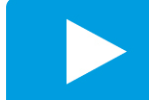

Watch 


\title{
Effects of mode structure on three-dimensional laser heating due to single or multiple rectangular laser beams
}

\author{
A. Kar \\ Mechanical \& Aerospace Engineering Department, Center for Research \& Education in Optics \& Lasers \\ (CREOL), University of Central Florida, 12424 Research Parkway, Suite 400, Orlando, Florida \\ 32826-3271 \\ J. E. Scott \\ Phillips Laboratory, Applied Laser Technology Branch (PL/LIDB), 3550 Aberdeen Avenue SE, Kirtland \\ AFB, New Mexico 87117-5776 \\ W. P. Latham \\ Phillips Laboratory, Lasers and Imaging Directorate (PL/LI), 3550 Aberdeen Avenue SE, Kirtland AFB, \\ New Mexico 87117-5776
}

(Received 9 November 1995; accepted for publication 8 April 1996)

\begin{abstract}
The natural shape of a typical chemical oxygen-iodine laser (COIL) beam is rectangular which means that the transverse cross section of the beam is rectangular. Such a rectangular beam can be split into two or more beams to carry out materials processing. The distance between such beams and the mode structure in the beams can affect the distribution of heat in the workpiece. This, in turn, affects the quality and efficiency of materials processing. A three-dimensional quasi-steady-state thermal model is presented in this article, and an expression for the temperature distribution during laser heating with single or multiple COIL beams having rectangular spots is obtained. Calculations are carried out for single or two rectangular COIL beams. The depth of heating is found to increase when the distance between two beams is less than a certain value.

(C) 1996 American Institute of Physics. [S0021-8979(96)02214-1]
\end{abstract}

\section{INTRODUCTION}

Since the development of high-power industrial lasers, it has been used in various types of materials processing such as cutting, welding, cladding. A fundamental understanding of the conversion of the optical energy of a laser beam into thermal energy and the propagation of the thermal energy in the workpiece is essential to utilize the laser technology efficiently and economically. The heat transfer phenomena depend on the size, shape, and mode structure of the laser beam at the substrate surface. Usually, the shape of the laser beam that comes out of certain types of resonators, such as chemical oxygen-iodine laser (COIL) and slab laser, is rectangular. Also, the laser beam is usually consisted of several $\mathrm{TEM}_{m n}$ modes. This article presents a mathematical model to understand the thermal effects due to mode structure of single or multiple rectangular laser beams.

\section{A. COIL development}

McDermott et al. ${ }^{1}$ invented the COIL in 1977 at the Air Force Weapons Laboratory which is now a part of the Phillips Laboratory. It is the shortest-wavelength, high-power chemical laser ever developed. The basic principle of this laser is based on the pumping of atomic iodine to its excited state, $\mathrm{I}\left({ }^{2} P_{1 / 2}\right)$, using resonant energy transfer from the metastable excited molecular oxygen state called singlet delta, or $\mathrm{O}_{2}\left({ }^{1} \Delta\right)$. Laser emission occurs at $1.315 \mu \mathrm{m}$ due to the deexcitation of the excited iodine to the ground state, $\mathrm{I}\left({ }^{2} P_{3 / 2}\right)$. Singlet delta oxygen is generated by inducing chemical reaction between gaseous chlorine $\left(\mathrm{Cl}_{2}\right)$ and an aqueous solution of hydrogen peroxide $\left(\mathrm{H}_{2} \mathrm{O}_{2}\right)$ and potassium hydroxide $(\mathrm{KOH})$.
Truesdell et $a .^{2}$ demonstrated the engineering scalability of a supersonic gas flow COIL with the $39 \mathrm{~kW}$ RotoCOIL device at the U.S. Air Force's Phillips Laboratory in 1989. This device used compact, rotating-disk, wetted-wall chemical reactors to generate large quantities of singlet delta oxygen, and then mixed the oxygen with iodine using a stacked supersonic nozzle array. ${ }^{3}$ Fujii et al. ${ }^{4-6}$ at Kawasaki Heavy Industries, Ltd., in Japan demonstrated the operation of a COIL for long durations with the operation of a $1 \mathrm{~kW}$ subsonic gas flow COIL for $2 \mathrm{~h}$. At the Phillips Laboratory, sustained high-power operation of a supersonic COIL was demonstrated with operation at $9 \mathrm{~kW}$ for $2 \min ^{7}$ and at 430 W for 13 min on a compact COIL test stand. ${ }^{8}$ Further details on COIL development and on the theoretical operation of COIL can be found Truesdell et al. ${ }^{2}$ and Avizonis and Truesdell, ${ }^{9}$ respectively.

Fujioka ${ }^{10}$ reviewed the transmission of a COIL beam through optical fibers in 1991 to examine the industrial applications of such beam delivery systems. Atsuta et al. ${ }^{11}$ and Fujii $^{12}$ at the Applied Laser Engineering Center (ALEC) in Japan demonstrated the transmission of a $1 \mathrm{~kW}$ COIL beam through a $300-\mu \mathrm{m}$-diam silica fiber at an intensity of 1.4 $\mathrm{MW} / \mathrm{cm}^{2}$.

\section{B. Heat conduction model development}

The conversion of the optical energy of a laser beam into thermal energy and the propagation of this thermal energy in the substrate play an important role in laser materials processing. A lot of work has been done to understand the heat conduction process during laser heating. Cline and Anthony ${ }^{13}$ solved the heat conduction equation using 
Green's function for a Gaussian beam moving at a constant velocity. They assumed constant thermophysical properties for the substrate, and that the substrate is semi-infinite in one direction and infinite in the other two directions. Lax ${ }^{14,15}$ analyzed the heat conduction process in a cylindrical medium due to a stationary Gaussian beam under steady state condition by considering both constant and temperaturedependent thermophysical properties for the substrate. In the case of temperature-dependent thermophysical properties, he solved the problem by linearizing it using Kirchhoff transformation. ${ }^{16}$ Hess et al. ${ }^{17}$ studied the temperature distribution in a radially infinite cylindrical medium by considering quasi-steady-state condition, temperature-dependent thermal conductivity, and neglecting the effect of the laser beam scanning velocity. Kokorowski, et al. ${ }^{18}$ examined the heat transfer process during $\mathrm{cw}$ laser annealing with a stationary beam beyond the melting temperature by solving the steadystate heat conduction equation in a radially infinite cylindrical medium with temperature-dependent thermal conductivity. Bell ${ }^{19}$ presented a one-dimensional model for laser annealing over a wide range of laser pulse durations and absorption coefficients. Nissim et al. ${ }^{20}$ analyzed the effects of elliptical or circular spot on the temperature distribution in semiconductors for a scanning cw laser beam. Moody and Hendel $^{21}$ solved the heat conduction equation by using a numerical technique to determine the temperature distribution in an infinite medium during laser heating. Kant ${ }^{22}$ studied the heating of a multilayered cylindrical medium resting on a half-space with a stationary laser beam. Modest and Abakians $^{23}$ examined the heat conduction in a moving semiinfinite solid under a pulsed, penetrating, Gaussian laser source. Kar and Mazumder ${ }^{24}$ presented a three-dimensional transient thermal model to determine the temperature distribution in finite slabs moving at a constant velocity by considering temperature-dependent thermophysical properties and laser irradiation with a Gaussian beam.

In all these studies, a single beam having a single mode, particularly $\mathrm{TEM}_{00}$ mode, was used. Kannatey-Asibu, ${ }^{25} \mathrm{Liu}$ and Kannatey-Asibu, ${ }^{26}$ and Chen and Kannatey-Asibu ${ }^{27}$ have examined the heat transfer phenomena due to laser heating with two Gaussian beams. Sharp et al. ${ }^{28}$ presented an analysis for the effect of mode structure on laser material processing by introducing a mode factor to describe the relative spot sizes of different modes. In most cases, the laser beam contains several modes. Also, the natural shape of the laser beam that comes out of the resonator of some special types of lasers, such as COIL and slab lasers, is rectangular. The purpose of this study is to develop a heat conduction model to understand the heating phenomena due to multimode, multiple rectangular laser beams.

\section{MATHEMATICAL MODEL}

The beam that comes out of a typical COIL cavity is rectangular in shape, and is consisted of several modes. The rectangular beam can be split into several beams. Such multiple beams provide an interesting feature to carry out materials processing, such as cutting, welding, cladding, etc., because it affects the temperature distribution differently compared to a single-beam heating phenomena. Also, the

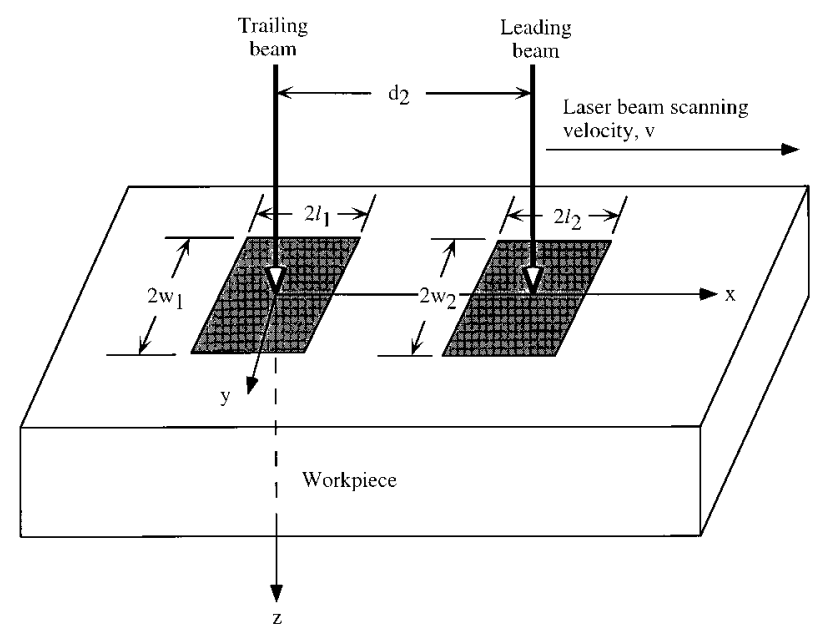

FIG. 1. Laser beam geometry and coordinate system for the mathematical model.

heating characteristics of a multimode beam is different from that of a Gaussian mode $\left(\mathrm{TEM}_{00}\right)$ beam. To understand the energy distribution in the workpiece during laser heating with rectangular, multimode, and single or multiple beams, a mathematical model is developed by considering the threedimensional quasi-steady-state heat conduction phenomena. Figure 1 shows the geometric configuration used in this model. The governing equation for the temperature distribution is given by

$$
\alpha_{e}\left(\frac{\partial^{2} T}{\partial x^{2}}+\frac{\partial^{2} T}{\partial y^{2}}+\frac{\partial^{2} T}{\partial z^{2}}\right)=-v \frac{\partial T}{\partial x}
$$

for $-\infty \leqslant x \leqslant \infty,-\infty \leqslant y \leqslant \infty$, and $0 \leqslant z \leqslant \infty$. Here $\alpha_{e}$ is the effective thermal diffusivity of the workpiece, which is defined by $\alpha_{e}=k /\left(\rho c_{\mathrm{pe}}\right)$, where $k$ and $c_{\mathrm{pe}}$ are the thermal conductivity and effective specific heat of the workpiece respectively. In this study, $c_{\mathrm{pe}}$ is taken to be $c_{\mathrm{pe}}=c_{p}+L_{m} / T_{m}$ to model the consumption of the latent heat of melting $L_{m}$, when the workpiece temperature reaches the melting temperature $T_{m}$. Here $c_{p}$ is the specific heat of the workpiece. $v$ is the velocity of the laser beam in the $x$ direction. All beams are assumed to move in the $x$ direction at a constant velocity $v$. Also, the centers of all beams are assumed to lie on the $x$ axis. Equation (1) is written in a moving Cartesian coordinate system whose origin lies at the center of one of the beams. The temperature $T$, which represents $T(x, y, z)$, in Eq.(1) is the dimensionless temperature of the workpiece, and is defined by

$$
T=\frac{T_{1}-T_{0}}{T_{m}-T_{0}},
$$

where $T_{1}, T_{0}$ are the actual and initial temperature of the substrate respectively.

The boundary conditions for Eq. (1) are given by

$$
\begin{array}{ll}
T \rightarrow 0 & \text { as } \quad x \rightarrow \pm \infty, \\
T \rightarrow 0 & \text { as } \quad y \rightarrow \pm \infty, \\
T \rightarrow 0 & \text { as } z \rightarrow \infty,
\end{array}
$$




$$
k \frac{\partial T}{\partial z}=-\frac{1}{T_{m}-T_{0}} I \quad \text { at } z=0 .
$$

Here the laser intensity $I$ is given by

$$
I=\sum_{s=1}^{S} \sum_{m=0}^{M} \sum_{n=0}^{N} I_{s, m n}
$$

where $S$ is the total number of beams used for heating the workpiece. $M$ and $N$ are the largest mode number of the beam in the $x$ and $y$ directions, respectively. $I_{s, m n}$ is the intensity of the $s$ th rectangular beam of $m$ th- and $n$ th-order modes, which is given by

$$
\begin{aligned}
I_{s, m n}= & \frac{2 A_{s} P_{s, m n}}{\pi 2^{m+n} m ! n ! l_{s 0} w_{s 0}} H_{m}^{2}\left(\frac{\sqrt{2}\left(x-d_{s}\right)}{l_{s 0}}\right) H_{n}^{2}\left(\frac{\sqrt{2} y}{w_{s 0}}\right) \\
& \times \exp \left(-\frac{2\left(x-d_{s}\right)^{2}}{l_{s 0}^{2}}\right) \exp \left(-\frac{2 y^{2}}{w_{s 0}^{2}}\right) .
\end{aligned}
$$

Here $P_{s, m n}$ is the total power of the $s$ th laser beam of $m$ thand $n$ th-order modes. $l_{s 0}$ and $w_{s 0}$ are, respectively, the half length and half width of the Gaussian mode of the sth beam. If $l_{s}$ and $w_{s}$ are, respectively, the half length and half width of the spot for the $s$ th beam with $\mathrm{TEM}_{m n}$ mode, $l_{s}$ and $w_{s}$ are related to Gaussian spot size by the expressions, $l_{s}$ $=l_{s 0} \sqrt{2 m+1}$ and $w_{s}=w_{s 0} \sqrt{2 n+1} \cdot d_{s}$ is the distance of the center of the $s$ th laser beam from the origin of the chosen coordinate system. In this study, the center of the first beam, trailing beam (see Fig. 1), coincides with the origin of the chosen coordinate system, and therefore, $d_{1}=0 . A_{s}$ is the absorptivity of the workpiece for the sth beam. $H_{m}$ and $H_{n}$ are, respectively, $m$ th and $n$th degree Hermite polynomials for the $m$ th and $n$th order modes of the laser beam. An ( $m$ +1 )th degree Hermite polynomial in $x$ can be written as

$$
H_{m+1}(x)=2 x H_{m}(x)-2 m H_{m-1}(x),
$$

where $H_{0}(x)=1$ and $H_{1}(x)=2 x$. To simplify Eq. (1), let us define

$$
T=T_{2} \exp (-b x),
$$

where

$$
b=\frac{v}{2 \alpha_{e}} .
$$

Substituting Eq. (6) into Eq. (1), we obtain

$$
\frac{\partial^{2} T_{2}}{\partial x^{2}}+\frac{\partial^{2} T_{2}}{\partial y^{2}}+\frac{\partial^{2} T_{2}}{\partial z^{2}}=-b^{2} T_{2} .
$$

The boundary conditions for $T_{2}$ are same as those given by the conditions (3a) $-(3 e)$. However, due to Eq. (6), boundary condition $(3 f)$ takes the following form:

$$
\frac{\partial T_{2}}{\partial z}=-\sum_{s=1}^{S} \sum_{m=0}^{M} \sum_{n=0}^{N} a_{m n, s} f_{m, s}(x) g_{n, s}(y) \quad \text { at } z=0,
$$

where

$$
a_{m n, s}=\frac{1}{k\left(T_{m}-T_{0}\right)} \frac{2 A_{s} P_{s, m n}}{\pi 2^{m+n} m ! n ! l_{s 0} w_{s 0}},
$$

$$
\begin{aligned}
& f_{m, s}(x)=H_{m}^{2}\left(\frac{\sqrt{2}\left(x-d_{s}\right)}{l_{s 0}}\right) \exp \left(b x-\frac{2\left(x-d_{s}\right)^{2}}{l_{s 0}^{2}}\right), \\
& g_{n, s}(y)=H_{n}^{2}\left(\frac{\sqrt{2} y}{w_{s 0}}\right) \exp \left(-\frac{2 y^{2}}{w_{s 0}^{2}}\right) .
\end{aligned}
$$

Using the following Fourier transform pair

integral transform:

$$
\begin{aligned}
\bar{T}_{2}(p, q, z)= & \int_{-\infty}^{\infty} d x \exp (i p x) \int_{-\infty}^{\infty} d y \\
& \times \exp (i q y) T_{2}(x, y, z),
\end{aligned}
$$

inversion formula:

$$
\begin{aligned}
T_{2}(x, y, z)= & \frac{1}{4 \pi^{2}} \int_{-\infty}^{\infty} d p \exp (-i p x) \int_{-\infty}^{\infty} d q \\
& \times \exp (-i q y) \bar{T}_{2}(p, q, z)
\end{aligned}
$$

the solution of Eq. (7) that satisfies the boundary condition (8) and the conditions as $x \rightarrow \pm \infty, y \rightarrow \pm \infty$, and $z \rightarrow \infty$, can be written as

$$
\begin{aligned}
T_{2}(x, y, z)= & \frac{1}{4 \pi^{2}} \sum_{s=1}^{S} \sum_{m=0}^{M} \sum_{n=0}^{N} a_{m n, s} \int_{-\infty}^{\infty} d p \\
& \times \exp (-i p x) \bar{f}_{m, s}(p) \int_{-\infty}^{\infty} d q \exp (-i q y) \\
& \times \bar{g}_{n, s}(q) \frac{\exp \left(-\sqrt{p^{2}+q^{2}+b^{2}} z\right)}{p^{2}+q^{2}+b^{2}},
\end{aligned}
$$

where $\bar{f}_{m, s}(p)$ and $\bar{g}_{n, s}(q)$ are Fourier transforms of $f_{m, s}(x)$ and $g_{n, s}(y)$, respectively, which are given by

$$
\begin{aligned}
& \bar{f}_{m, s}(p)=\int_{-\infty}^{\infty} d x \exp (\text { ipx }) f_{m, s}(x), \\
& \bar{g}_{n, s}(q)=\int_{-\infty}^{\infty} \exp (\text { iqy }) g_{n, s}(y) .
\end{aligned}
$$

Noting that the Fourier inverse transform $\left(\mathscr{F}^{-1}\right)$ of [exp $\left.\left(-\sqrt{p^{2}+q^{2}+b^{2} z}\right)\right] / \sqrt{p^{2}+q^{2}+b^{2}}$ is given by

$$
\begin{aligned}
\mathscr{T}-1 & \left\{\frac{\exp \left(-\sqrt{p^{2}+q^{2}+b^{2}} z\right)}{\sqrt{p^{2}+q^{2}+b^{2}}}\right\} \\
= & \frac{1}{4 \pi^{2}} \int_{-\infty}^{\infty} d p \exp (-i p x) \int_{-\infty}^{\infty} d q \\
& \times \exp (-i q y) \frac{\exp \left(-\sqrt{p^{2}+q^{2}+b^{2}} z\right)}{\sqrt{p^{2}+q^{2}+b^{2}}} \\
= & \frac{1}{2 \pi} \frac{\exp \left(-b \sqrt{r^{2}+z^{2}}\right)}{\sqrt{r^{2}+z^{2}}},
\end{aligned}
$$

where $r=\sqrt{x^{2}+y^{2}}$, and applying the convolution theorem to Eq. (11), we can obtain an expression for $T_{2}(x, y, z)$ which can be substituted into Eq. (6) to obtain the following expression for the dimensionless temperature $T(x, y, z)$. 
TABLE I. Typical values for the thermophysical properties of iron. Due to the lack of high-temperature data, these values were use din this study to obtain various results although the substrate was 400 series stainless steel.

\begin{tabular}{ll}
\hline \multicolumn{1}{c}{ Thermophysical properties } & Values of thermophysical properties \\
\hline Density, $\rho$ & $7870 \mathrm{~kg} \mathrm{~m}^{-3}$ \\
Melting point, $T_{m}$ & $1809 \mathrm{~K}$ \\
Boiling point, $T_{b}$ & $3133 \mathrm{~K}$ \\
Thermal conductivity, $k$ & $78.2 \mathrm{~W} \mathrm{~m}^{-1} \mathrm{~K}^{-1}$ \\
Specific heat, $c_{p}$ & $456 \mathrm{~J} \mathrm{~kg}^{-1} \mathrm{~K}^{-1}$ \\
Effective specific heat, $c_{\mathrm{pe}}=c_{p}+L_{m} / T_{m}$ & $606 \mathrm{~J} \mathrm{~kg}^{-1} \mathrm{~K}^{-1}$ \\
Thermal diffusivity, $\alpha=k /\left(\rho c_{p}\right)$ & $2.18 \times 10^{-5} \mathrm{~m}^{2} \mathrm{~s}^{-1}$ \\
Effective thermal diffusivity, $\alpha_{e}=k /\left(\rho c_{\mathrm{pe}}\right)$ & $1.64 \times 10^{-5} \mathrm{~m}^{2} \mathrm{~s}^{-1}$ \\
Latent heat of melting, $L_{m}$ & $2.72 \times 10^{5} \mathrm{~J} \mathrm{~kg}^{-1}$ \\
Latent heat of boiling, $L_{b}$ & $6.10 \times 10^{6} \mathrm{~J} \mathrm{~kg} \mathrm{k}^{-1}$ \\
\hline \hline
\end{tabular}

$$
\begin{aligned}
T(x, y, z)= & \frac{\exp (-b x)}{\pi} \sum_{s=1}^{S} \sum_{m=0}^{M} \sum_{n=0}^{N} a_{m n, s} \\
& \times \int_{0}^{\infty} r^{\prime} d r^{\prime} f_{m, s}\left(x-r^{\prime} \cos \theta\right) \\
& \times \int_{0}^{\pi} d \theta g_{n, s}\left(y-r^{\prime} \sin \theta\right) \\
& \times \frac{\exp \left(-b \sqrt{r^{\prime 2}+z^{2}}\right)}{\sqrt{r^{\prime 2}+z^{2}}} .
\end{aligned}
$$

The integrals in Eq. (14) are numerically evaluated by using the trapezoidal rule for numerical integration to calculate the temperature distribution for various process parameters using typical values for the thermophysical properties $\left(\right.$ Brandes $^{29}$ ) of steel as given in Table I.

\section{RESULTS AND DISCUSSION}

The temperature field, $T(x, y, z)$, obtained from Eq. (14) is discussed in this section to analyze the heating phenomena due to rectangular, multimode, and single or multiple laser beams. The effects of various process parameters such as the laser mode $\left(\mathrm{TEM}_{m n}\right)$ structure, distance between the two beams in the case of two-beam materials processing, shape and size of the laser spot, and scanning velocity are examined. In this study, $M$ and $N$ are arbitrarily chosen to be 3 and 1 , respectively, just to compute $T(x, y, z)$. It should be noted that $M=3$ and $N=1$ correspond to $m=0,1,2,3$ and $n=0,1$ respectively. The mode numbers and laser power distributions used in this study for various figures are listed in Table II. The absorptivity of the substrate $\left(A_{s}\right.$, for $s=1,2, \ldots, S)$ is taken to be $15 \%$ in all cases.

Figures 2 and 3 represent three-dimensional views of the intensity and temperature profiles, respectively, for a beam having $m=0,1,2,3$ and $n=0,1$ modes which means that the beam has 4 and 2 modes in the $x$ and $y$ directions, respectively. Due to the particular choice of the power distribution in each mode given by Table II, the intensity is low at the beam center and it becomes maximum near the edges of the beam in both $x$ and $y$ directions as shown in Fig. 2. Figure 3 represents the temperature distribution, $T(x, y, 0)$ at the substrate surface where the origin of the coordinate system is located at the beam center. Due to the motion of the laser beam in the positive $x$ direction relative to the substrate, convection heat flow occurs in the negative $x$ direction which causes the temperature to be lower in front of the beam than at the back of the beam.

Figures 4 and 5 show the substrate surface temperature

\begin{tabular}{|c|c|c|c|c|c|c|c|c|c|}
\hline \multirow[b]{2}{*}{ Figures } & \multirow[b]{2}{*}{$n$} & \multicolumn{4}{|c|}{$\begin{array}{l}\text { Power distribution in various modes } \\
\text { of the trailing beam, } P_{1}(\mathrm{~kW}) \\
m\end{array}$} & \multicolumn{4}{|c|}{$\begin{array}{l}\text { Power distribution in various modes } \\
\text { of the leading beam, } P_{2}(\mathrm{~kW}) \\
m\end{array}$} \\
\hline & & 0 & 1 & 2 & 3 & 0 & 1 & 2 & 3 \\
\hline \multirow[t]{2}{*}{ Figs. $2,3,6,10,11,12,13$} & 0 & 0.1 & 0.4 & 1.0 & 1.5 & & & & \\
\hline & 1 & 0.1 & 0.4 & 1.0 & 1.5 & & & & \\
\hline \multirow[t]{4}{*}{ Figs. 7,8} & 0 & 0.05 & 0.2 & 0.5 & 0.75 & 0.05 & 0.2 & 0.5 & 0.75 \\
\hline & 1 & 0.05 & 0.2 & 0.5 & 0.75 & 0.05 & 0.2 & 0.5 & 0.75 \\
\hline & 0 & 0.017 & 0.067 & 0.17 & 0.25 & 0.085 & 0.335 & 0.85 & 1.25 \\
\hline & 1 & 0.017 & 0.067 & 0.17 & 0.25 & 0.085 & 0.335 & 0.85 & 1.25 \\
\hline \multirow[t]{4}{*}{ Fig. 9} & 0 & 0.05 & 0.2 & 0.5 & 0.75 & 0.05 & 0.2 & 0.5 & 0.75 \\
\hline & 1 & 0.05 & 0.2 & 0.5 & 0.75 & 0.05 & 0.2 & 0.5 & 0.75 \\
\hline & 0 & 0.085 & 0.335 & 0.85 & 1.25 & 0.017 & 0.067 & 0.17 & 0.25 \\
\hline & 1 & 0.085 & 0.335 & 0.85 & 1.25 & 0.017 & 0.067 & 0.17 & 0.25 \\
\hline
\end{tabular}
distributions for one and two Gaussian mode $\left(\mathrm{TEM}_{00}\right)$ beams, respectively. The length and width of the laser spot in

TABLE II. Power distribution data used in this study for various $\mathrm{TEM}_{m n}$ modes. 


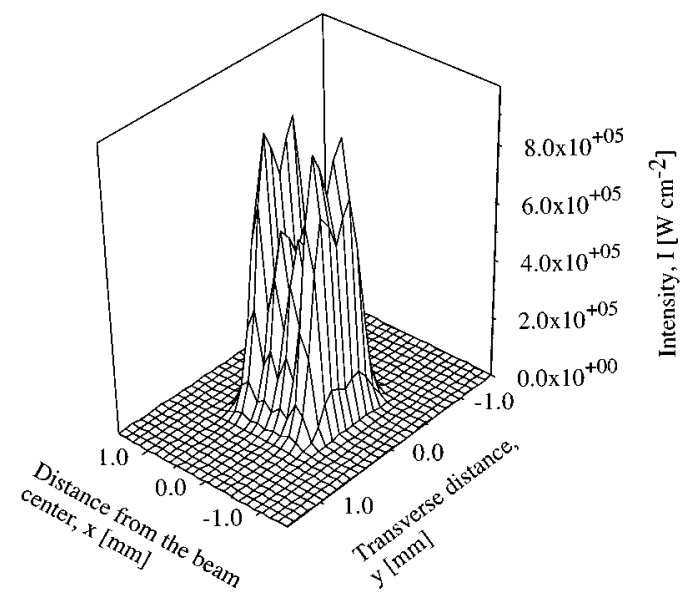

FIG. 2. Three-dimensional view of the intensity profile for a single multimode $(m=0,1,2,3$ and $n=0,1)$ beam. $P=6 \mathrm{~kW}, 2 l_{1}=1.7 \mathrm{~mm}, 2 w_{1}=1.2$ $\mathrm{mm}$, and $v=1 \mathrm{~cm} / \mathrm{s}$.

Fig. 4 is 1.7 and $1.2 \mathrm{~mm}$, respectively, and the total power is $6 \mathrm{~kW}$. In Fig. 5, where two beams are used, the length and width of the spot of each beam are 0.85 and $1.2 \mathrm{~mm}$, respectively, and the power of each beam is $3 \mathrm{~kW}$. These two beams are arranged in such a way that the front edge of the first beam, which is referred to as the trailing beam in Fig. 1, coincides with the rear edge of the second beam, which is referred to as the leading beam in Fig. 1. Although the laser intensity is the same in both figures, the peak temperature in the case of a single beam is higher than in the case of two beams because the intensity is confined in a region of length $1.7 \mathrm{~mm}$ in Fig. 4, whereas it is confined in two regions of length $0.85 \mathrm{~mm}$ each in Fig. 5. The intensity is very low in an area near $x=0.425 \mathrm{~mm}$ where the boundaries of these two regions meet, and therefore, heat is conducted into this area of low intensity to raise the substrate temperature as shown in Fig. 5. Due to this, two beams heat up a larger area than a single beam does, which causes the peak temperature to rise

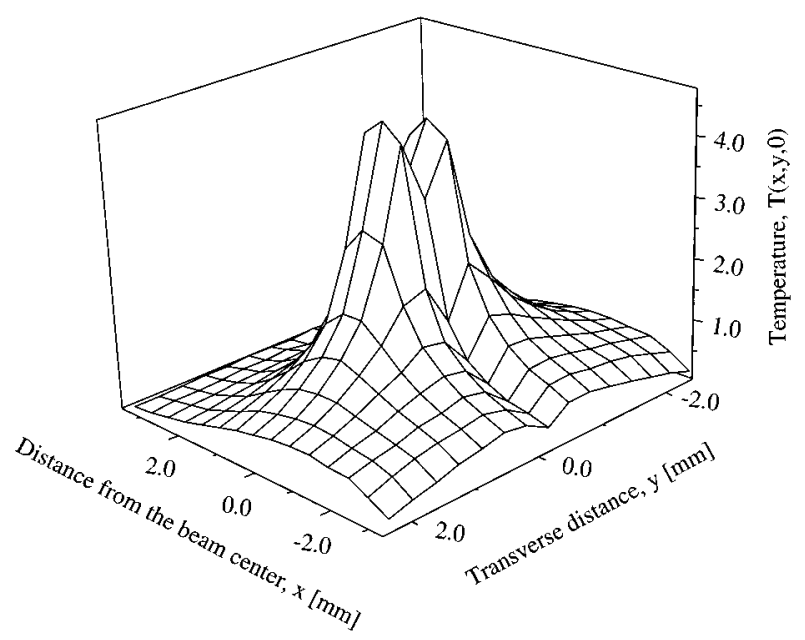

FIG. 3. Three-dimensional view of the normalized surface temperature profile for a single multimode $(m=0,1,2,3$ and $n=0,1)$ beam. $P=6 \mathrm{~kW}$, $2 l_{1}=1.7 \mathrm{~mm}, 2 w_{1}=1.2 \mathrm{~mm}$, and $v=1 \mathrm{~cm} / \mathrm{s}$.

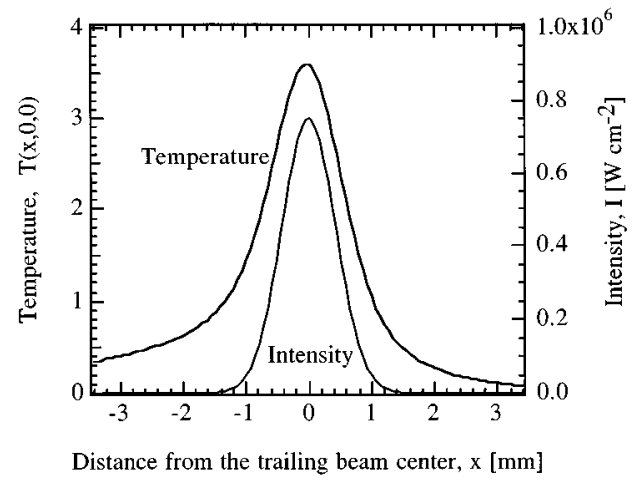

FIG. 4. Normalized surface temperature and intensity profiles along the $x$ axis $(y=0$ and $z=0)$ for a single Gaussian mode $\left(\mathrm{TEM}_{00}\right)$ beam. $P=6 \mathrm{~kW}$, $2 l_{1}=1.7 \mathrm{~mm}, 2 w_{1}=1.2 \mathrm{~mm}$, and $v=1 \mathrm{~cm} / \mathrm{s}$.

less in the former case than in the latter case, even though the substrate surface areas irradiated by the laser are the same in both cases.

Figures 6 and 7 are based on a laser having $m=0,1,2,3$ and $n=0,1$ modes. Due to the particular choice of the modes and power distribution in each mode, the intensity has $M$-shaped distribution in the $x$ direction as shown in Figs. 6 and 7. The peak temperature in Fig. 7 is lower than in Fig. 6 for the reason explained in the context of Figs. 4 and 5. Also, the temperature is lower in front of the beam than at the back of the beam because heat is convected away towards the back of the beam (negative $x$ direction) due to the motion of the substrate in the negative $x$ direction relative to the beam.

Figure 8 shows the effects of the distance between the two beams on the surface temperature distribution for two beams having modes $m=0,1,2,3$ and $n=0,1$. The spot length and power of each beam are $0.85 \mathrm{~mm}$ and $3 \mathrm{~kW}$, respectively. Curves 1,2 , and 3 represent three physical situations of overlapping $\left(d_{2}=0.425 \mathrm{~mm}\right)$, touching $\left(d_{2}=0.825 \mathrm{~mm}\right)$, and disjoint $\left(d_{2}=1.7 \mathrm{~mm}\right)$ spots, respectively, where $d_{2}$ is the distance between the centers of the two beams. As $d_{2}$ decreases, the size of the laser-heated region decreases, which means that less amount of material will be heated up by the beam, and, therefore, the peak temperature will increase as shown in Fig. 8.

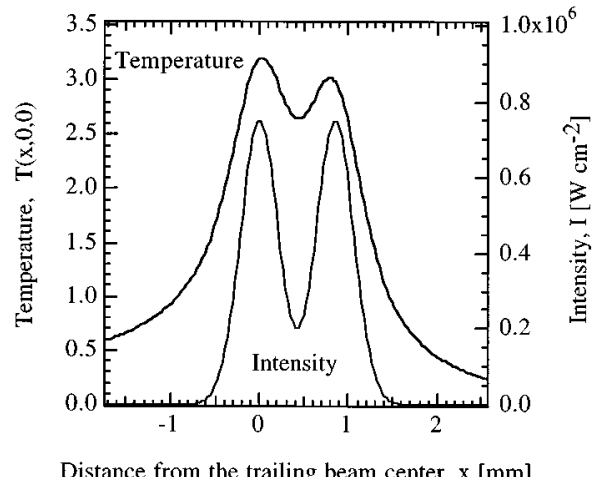

FIG. 5. Normalized surface temperature and intensity profiles along the $x$ axis $(y=0$ and $z=0)$ for two Gaussian mode $\left(\right.$ TEM $\left._{00}\right)$ beams. $P_{i}=3 \mathrm{~kW}$, $2 l_{i}=0.85 \mathrm{~mm}, 2 w_{i}=1.2 \mathrm{~mm}$ for $i=1$ and $2, v=1 \mathrm{~cm} / \mathrm{s}$, and $d_{2}=0.85 \mathrm{~mm}$. 


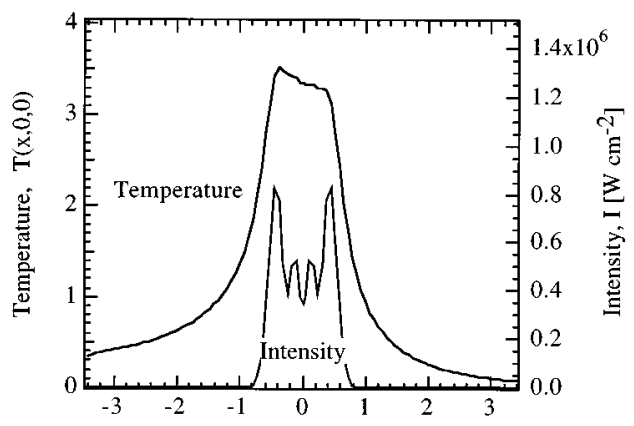

Distance from the trailing beam center, $x[\mathrm{~mm}]$

FIG. 6. Normalized surface temperature and intensity profiles along the $x$ axis $(y=0$ and $z=0)$ for a single multimode $(m=0,1,2,3$ and $n=0,1)$ beam. $P=6 \mathrm{~kW}, 2 l_{1}=1.7 \mathrm{~mm}, 2 w_{1}=1.2 \mathrm{~mm}$, and $v=1 \mathrm{~cm} / \mathrm{s}$.

Figure 9 examines the effects of the power content in each beam on the surface temperature profile. The length of each beam is $0.85 \mathrm{~mm}$, and the centers of the two beams are $0.85 \mathrm{~mm}$ apart. The powers of each beam given in Fig. 9 show that the powers in the trailing beams for curves 1,2 , and 3 are 1,3 , and $5 \mathrm{~kW}$, respectively. These three curves indicate that high peak temperature is attained near the beam that has higher power relative to the other beam. The peak temperatures $(T)$ for curves 3,2 , and 1 are 4.33, 3.22, and 4.30 , respectively. Since the power contents in the trailing and leading beams are reversed for curves 3 and 1, the peak temperatures for these two cases would be identical due to the conduction effect alone. However, the peak temperature for curve 3 is higher than that for curve 1 is due to the convection heat flow in the negative $x$ direction. These two curves suggest that the power contents of the two beams have to be chosen properly to attain a high peak temperature by taking advantage of the convection heat flow due to the substrate motion relative to the beam.

Figures 10 and 11 show the temperature variations along the $x$ axis for various lateral $(y)$ and axial $(z)$ locations, respectively. It can be seen from Fig. 10 that the temperature is very high (see curve 1) near the edge of the beam due to the bimodal structure of the beam modes $(m=0,1,2,3$ and $n=0,1)$ in the $y$ direction. But it decreases sharply as one

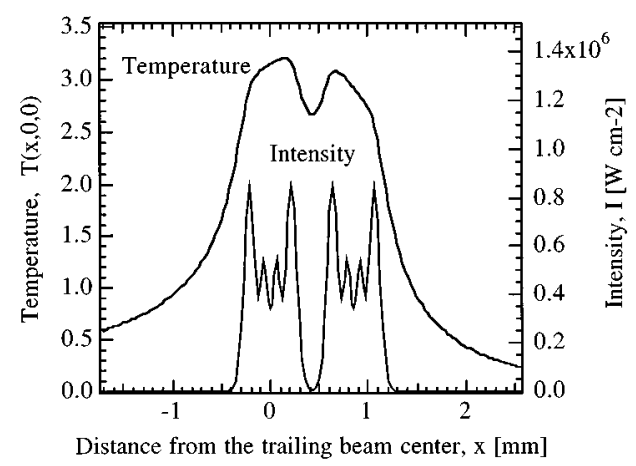

FIG. 7. Normalized surface temperature and intensity profiles along the $x$ axis $(y=0$ and $z=0)$ for two multimode $(m=0,1,2,3$ and $n=0,1)$ beams. $P_{i}=3 \mathrm{~kW}, 2 l_{i}=0.85 \mathrm{~mm}, 2 w_{i}=1.2 \mathrm{~mm}$ for $i=1$ and $2, v=1 \mathrm{~cm} / \mathrm{s}$, and $d_{2}=0.85 \mathrm{~mm}$.

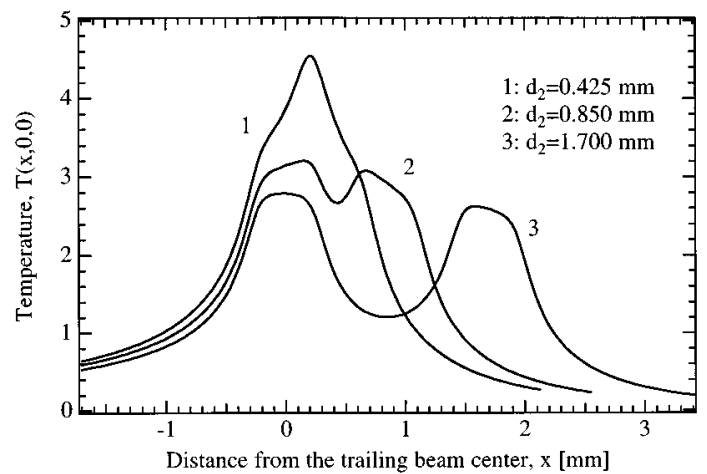

FIG. 8. Effects of the distance between two beams on the normalized surface temperature profile along the $x$ axis $(y=0$ and $z=0)$ for two multimode ( $m=0,1,2,3$ and $n=0,1$ ) beams. $P_{i}=3 \mathrm{~kW}, 2 l_{i}=0.85 \mathrm{~mm}, 2 w_{i}=1.2 \mathrm{~mm}$ for $i=1$ and $2, v=1 \mathrm{~cm} / \mathrm{s}$.

moves away from the beam. Figure 11 shows that the temperature is very high near the surface and that it decreases sharply as one moves deeper into the substrate. By analyzing these types of results concerning the temperature distribution, one can calculate the size of the heat-affected zone to determine the thermodynamic and metallurgical effects of the laser materials processing.

Figure 12 represents the surface temperature distribution along the $x$ axis for various values of the laser beam scanning velocity for a single multimode $(m=0,1,2,3$ and $n=0,1)$ beam. It should be noted that the temperature is lower in front of the beam than at the back of the beam due to the convection heat flow in the negative $x$ direction, which is indicated by curve 3 for which the scanning velocity is 1 $\mathrm{cm} / \mathrm{s}$. As the scanning velocity decreases, the effect of the convection heat flow decreases and the conduction heat flow becomes dominant, and therefore, the temperature distribution tends to be symmetric as shown by curves 2 and 1 .

Figure 13 shows the effects of the spot shape, that is the aspect ratio $a=l_{1} / w_{1}$, on the surface temperature distribution for a single multimode $(m=0,1,2,3$ and $n=0,1)$ beam. The widths of the spots for curves 1,2 , and 3 are $3.2,1.428$, and $0.82 \mathrm{~mm}$, respectively. The aspect ratios for curves 1,2 ,

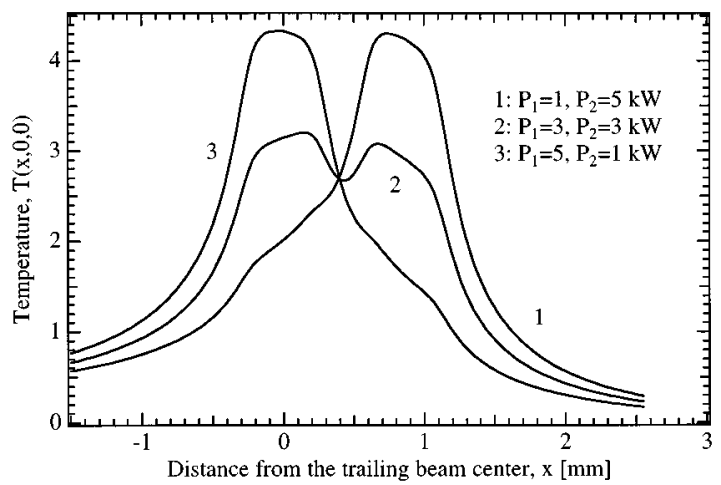

FIG. 9. Effects of the power content in two beams on the normalized surface temperature profile along the $x$ axis $(y=0$ and $z=0)$ for two multimode ( $m=0,1,2,3$ and $n=0,1$ ) beams. $2 l_{i}=0.85 \mathrm{~mm}, 2 w_{i}=1.2 \mathrm{~mm}$ for $i=1$ and 2 , $v=1 \mathrm{~cm} / \mathrm{s}$, and $d_{2}=0.85 \mathrm{~mm}$. 


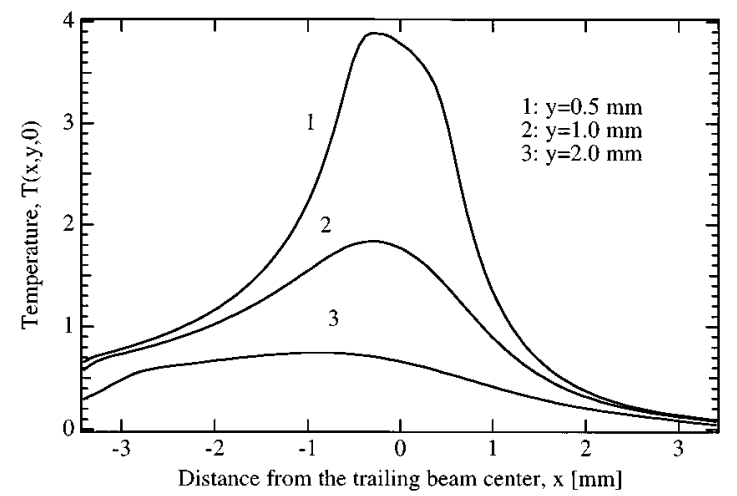

FIG. 10. Normalized temperature distribution along the $x$ axis for various valus of $y$ when $z=0$. A single multimode $(m=0,1,2,3$ and $n=0,1)$ beam is used. $P=6 \mathrm{~kW}, 2 l_{1}=1.7 \mathrm{~mm}, 2 w_{1}=1.2 \mathrm{~mm}$, and $v=1 \mathrm{~cm} / \mathrm{s}$.

and 3 are $0.2,1$, and 3, respectively, which make the spot area to be about $2 \mathrm{~mm}^{2}$ for these three curves. It can be seen from Fig. 13 that the peak temperature is higher for curve 2 than those for curves 1 and 3. This is because the surface area per unit length of a parallelepiped with a square cross section is lower than that with a rectangular cross section. Since heat loss is directly proportional to the surface area, less heat is lost for the case of curve 2 for which the spot is a square. Therefore, more energy is utilized for the case of curve 2 to heat up the material than for the cases of curves 1 and 3 for which the spots are rectangular.

\section{CONCLUSIONS}

A three-dimensional quasi-steady-state heat conduction model is presented for the analysis of laser heating with rectangular or square, multimode, and single or multiple laser beams. The heat conduction problem is solved using the Fourier transform to obtain an integral expression for the temperature field. The integrals of this expression are evaluated numerically by using the trapezoidal rule for numerical integration.

Due to the particular choice of the beam modes $(\mathrm{m}$ $=0,1,2,3$ and $n=0,1)$ in this study, the laser intensity $I$ is found to have an $M$-shaped nonuniform profile. However,

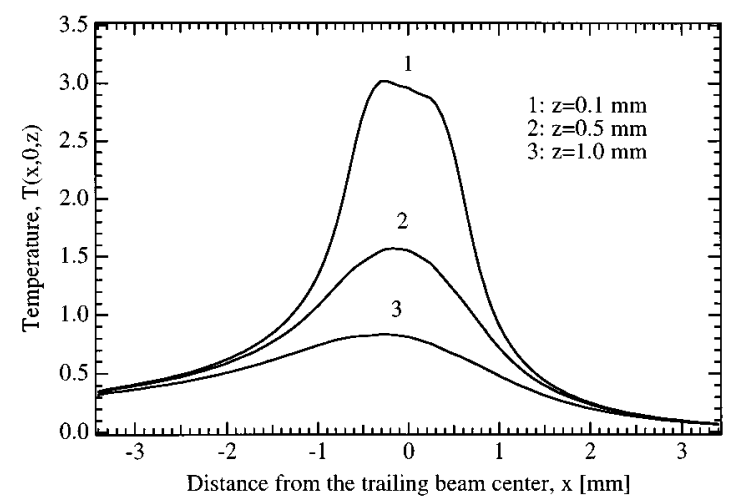

FIG. 11. Normalized temperature distribution along the $x$ axis for various values of $z$ when $y=0$. A single multimode $(m=0,1,2,3$ and $n=0,1)$ beam is used. $P=6 \mathrm{~kW}, 2 l_{1}=1.7 \mathrm{~mm}, 2 w_{1}=1.2 \mathrm{~mm}$, and $v=1 \mathrm{~cm} / \mathrm{s}$.

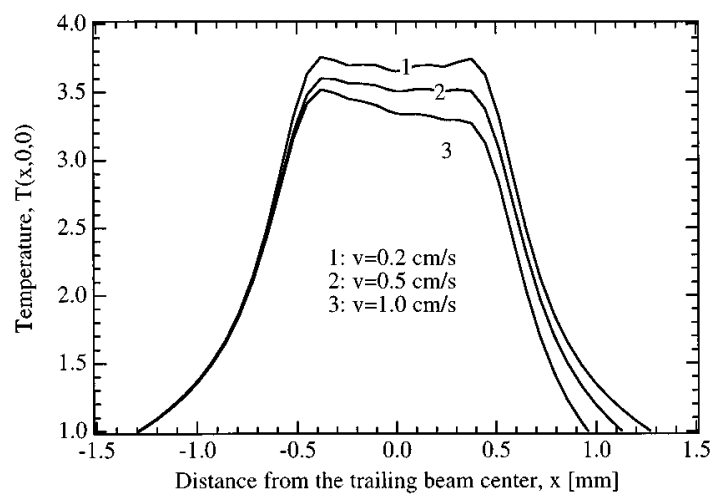

FIG. 12. Effects of the laser beam scanning velocity on the normalized surface temperature distribution along the $x$ axis $(y=0$ and $z=0)$. A single multimode $(m=0,1,2,3$ and $n=0,1)$ beam is used. $P=6 \mathrm{~kW}, 2 l_{1}=1.7 \mathrm{~mm}$, $2 w_{1}=1.2 \mathrm{~mm}$.

the substrate surface temperature during laser heating with a single beam having such a nonuniform intensity profile is found to increase steadily from the front edge of the beam to the rear edge of the beam in the laser spot region. The temperature at the back of the beam is higher than in front of the beam because of the convection heat flow due to the motion of the substrate towards the back of the beam. When the laser scanning velocity, that is the relative motion between the substrate and laser beam, decreases, the temperature field tends towards a uniform distribution in the laser spot region.

The intensity of each beam is found to have an interesting effect on the peak temperature during laser heating with multiple beams. For a given scanning velocity, the peak temperature in the trailing laser spot region is higher than or equal to that in the leading laser spot region if the intensity of the trailing beam is higher than or equal to that of the leading beam. However, the peak temperature in the trailing laser spot region can be lower than that in the leading laser spot region if the intensity of the trailing beam is lower than that of the leading beam in spite of the convection heat flow towards to the trailing laser spot region. The shape of the laser spot also has an interesting effect on the peak temperature in the laser spot region during laser heating with a single beam. The peak temperature is found to be higher for a

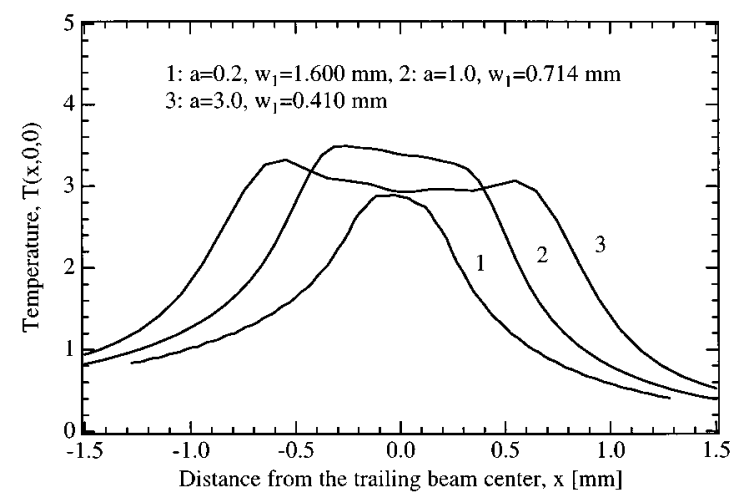

FIG. 13. Effects of the aspect ratio $\left(a=l_{1} / w_{1}\right)$ on the normalized surface temperature distribution along the $x$ axis $(y=0$ and $z=0)$. A single multimode $(m=0,1,2,3$ and $n=0,1)$ beam is used. $P=6 \mathrm{~kW}$ and $v=1 \mathrm{~cm} / \mathrm{s}$. 
square spot than for rectangular spots for the same laser intensity and material processing conditions. This is because of less energy loss for a square spot than for rectangular spots, which means that the energy utilization is improved if a square laser spot is used.

\section{ACKNOWLEDGMENTS}

This work was accomplished when A. Kar was at the Phillips Laboratory, PL/LIDB, in the Kirtland Air Force Base under the 1995 summer faculty research Program sponsored by the Air Force Office of Scientific Research (AFOSR). Testing was contributed by the Applied Laser Technology Branch of the Phillips Laboratory.

${ }^{1}$ W. E. McDermott, N. R. Pchelkin, D. J. Benard, and R. R. Bousek, Appl. Phys. Lett. 32, 469 (1978).

${ }^{2}$ K. A. Truesdell, C. A. Helms, and G. D. Hager, "COIL Development in the USA," 25th Plasmadynamics and Lasers Conference, AIAA 94-2421, June 1994

${ }^{3}$ J. E. Scott, J. L. R. Shaw, K. A. Truesdell, G. D. Hager, and C. A. Helms, "Design Considerations for the Chemical Oxygen-Iodine Supersonic Mixing Nozzle," 25th Plasmadynamics and Lasers Conference, AIAA 94-2436, 1994.

${ }^{4}$ H. Fujii, S. Yoshida, M. Iizuka, and T. Atsuta, J. Appl. Phys. 66, 1033 (1989).

${ }^{5}$ F. Wani, N. Naitou, T. Nagai, M. Iizuka, H. Tsuji, and H. Fujii, Proceedings of Laser Advanced Materials Processing (LAMP '92), Niigata, Japan, June 1992, pp. 127-132.

${ }^{6} \mathrm{H}$. Fujii and T. Atsuta, "Current Status of Industrial COIL Development," Third International Workshop on Iodine Lasers and Applications, Bechyne Castle, Czechoslovakia, 1992, SPIE Vol. 1980, pp. 148-152.

${ }^{7}$ K. A. Truesdell, T. Lonergan, C. Wisniewski, K. Healey, J. Scott, and C. Helms, "COIL Thermal Management,'” 25th Plasmadynamics and Lasers Conference, AIAA 94-2441, June 1994.

${ }^{8}$ S. Phipps, C. A. Helms, and K. A. Truesdell, "Compact CW Supersonic Chemical Oxygen Iodine Laser (COIL)," 25th Plasmadynamics and Lasers Conference, AIAA 94-2453, June 1994.

${ }^{9}$ P. V. Avizonis and K. A. Truesdell, "Historical Perspectives of the
Chemical Oxygen-Iodine Laser (COIL),' 25th Plasmadynamics and Lasers Conference, AIAA 94-2416, June 1994.

${ }^{10}$ T. Fujioka, Infrared Phys. 32, 81 (1991).

${ }^{11}$ T. Atsuta, K. Yasuda, T. Matsumoto, T. Sakurai, and H. Okado, in Conference on Lasers and Electro-Optics (CLEO) '94, Vol. 8, 1994 OSA Technical Digest Series (Optical Society of America, Washington, DC, 1994), p. 351.

${ }^{12}$ H. Fujii, "COIL Development in Japan," 25th Plasmadynamics and Lasers Conference, AIAA 94-2410, June 1994.

${ }^{13}$ H. E. Cline and T. R. Anthony, J. Appl. Phys. 48, 3895 (1977).

${ }^{14}$ M. Lax, J. Appl. Phys. 48, 3919 (1977).

${ }^{15}$ M. Lax, J. Appl. Phys. Lett. 33, 786 (1978).

${ }^{16}$ H. S. Carslaw and J. C. Jaeger, Conduction of Heat in Solids, 2nd ed. (Clarendon, Oxford, 1986), pp. 10-11.

${ }^{17}$ L. D. Hess, R. A. Forber, S. A. Kokorowski, and G. L. Olson, in Proceedings of the Society of Photo-Optical Instrumentation Engineers, edited by J. F. Ready (Society of Photo-Optical Instrumentation Engineers, Washington, DC, 1980), Vol. 198, pp. 31-34.

${ }^{18}$ S. A. Kokorowski, G. L. Olson, and L. D. Hess, in Proceedings of Laser and Electron-Beam Solid Interactions and Materials Processing, edited by J. F. Gibons, L. D. Hess, and T. W. Sigman (Elsevier, North Holland, 1985), pp. 139-146.

${ }^{19}$ A. E. Bell, RCA Rev. 40, 295 (1979).

${ }^{20}$ Y. I. Nissim, A. Lietoila, R. B. Gold, and J. F. Gibons, J. Appl. Phys. 51, 274 (1980).

${ }^{21}$ J. E. Moody and R. H. Hendel, J. Appl. Phys. 53, 4364 (1982).

${ }^{22}$ R. Kant, J. Appl. Mech. 55, 93 (1988).

${ }^{23}$ M. F. Modest and H. Abakians, J. Heat Transfer 108, 597 (1986).

${ }^{24}$ A. Kar and J. Mazumder, J. Appl. Phys. 65, 2923 (1989).

${ }^{25}$ E. Kannatey-Asibu, J. Eng. Mater. Tech. 113, 215 (1991).

${ }^{26}$ Y. N. Liu and E. Kannatey-Asibu, J. Heat Transfer 115, 34 (1993).

${ }^{27}$ T. C. Chen and E. Kannatey-Asibu, in Proceedings of the International Congress on the Applications of Lasers and Electro-Optics, 1994 (ICALEO '94): Laser Materials Processing, edited by T. D. McCay, A. Matsunawa, and H. Hugel (Laser Institute of America, Florida, 1994), Vol. 2500, pp. 668-677.

${ }^{28}$ M. Sharp, P. Henry, W. M. Steen, and G. C. Lim, in Proceedings of the 6th International Congress, Laser '83 Optoelectronics: Optoelectronics in Engineering, edited by W. Waidelich (Springer, Berlin, 1984), pp. 243246.

${ }^{29}$ Smithells Metals Reference Book, edited by E. A. Brandes, 6th ed. (Butterworths, London, 1983), pp. 8-2 and 14-1. 